University of Nebraska - Lincoln

DigitalCommons@University of Nebraska - Lincoln

Timothy J. Gay Publications

Research Papers in Physics and Astronomy

$11-2020$

\title{
Helmholtz spacing of thin rectangular magnetic field coils
}

Karl J. Ahrendsen

Sarah Reyes

Timothy J. Gay

Follow this and additional works at: https://digitalcommons.unl.edu/physicsgay

Part of the Atomic, Molecular and Optical Physics Commons, and the Other Physics Commons

This Article is brought to you for free and open access by the Research Papers in Physics and Astronomy at DigitalCommons@University of Nebraska - Lincoln. It has been accepted for inclusion in Timothy J. Gay Publications by an authorized administrator of DigitalCommons@University of Nebraska - Lincoln. 


\title{
Helmholtz spacing of thin rectangular magnetic field coils
}

\author{
Cite as: Rev. Sci. Instrum. 91, 116103 (2020); doi: 10.1063/5.0023024 \\ Submitted: 27 July 2020 - Accepted: 13 October 2020 • \\ Published Online: 3 November 2020
}

K. J. Ahrendsen, a) (D) S. Reyes, (D) and T. J. Gay

AFFILIATIONS

Jorgensen Hall, University of Nebraska, Lincoln, Nebraska 68588-0299, USA

a) Author to whom correspondence should be addressed: karl@huskers.unl.edu

\begin{abstract}
In this Note, we discuss the Helmholtz spacing for a pair of thin rectangular coils of arbitrary aspect ratio and consider how best to use such coils to compensate for Earth's magnetic field along the coils' Cartesian symmetry axes. Such coils are frequently used in conjunction with charged-particle beam machines. The Helmholtz spacing varies non-monotonically between that for square coils and that for four optimally spaced infinite wires. We consider other coil spacings that extend the length over which the field varies by less than some tolerance along the Cartesian symmetry axes. The calculations also provide a convenient means to evaluate when the length of the coils is sufficiently long to be considered infinite at the center point within a fixed tolerance.
\end{abstract}

Published under license by AIP Publishing. https://doi.org/10.1063/5.0023024

A variety of "table-top" apparatus used in physics comprise a source of charged particles, an experimental target chamber, and a beam-transport line connecting the two. To minimize the deflection of the charged particle beam by magnetic fields from the Earth and other sources, the "beamline" should, if possible, be aligned with the horizontal component of the local field. The vertical component can then be minimized by one or more current-carrying coils surrounding the entire apparatus. The most common arrangement for doing this involves a pair of circular coils of radius $r$, which are separated along their mutual normal axis by $r$. This "Helmholtz" separation, $S_{H}$, results in a field along the normal axis which has no second spatial derivative along that axis midway between the coils. The first derivative is zero by virtue of symmetry, and this leads to an extended region of a relatively uniform field.

In some experimental arrangements in which the distance between the charged particle source and the experimental chamber is comparable to or larger than the target chamber, a pair of rectangular coils may prove to be the best option for "zeroing out" the vertical component of the field both along the beamline and in the target volume. The general topic of the field produced by two rectangular coils has been taken up by a number of authors, ${ }^{1-3}$ but, to our knowledge, no one has discussed systematically the analog of $S_{H}$ for a set of rectangle coils of arbitrary dimensions. We use the same criteria of a null second spatial derivative as the characteristic, which defines the Helmholtz spacing. In this Note, we identify such a spacing and also consider other spacings that extend the length of the uniform field, within a tolerance, $T$, along the three Cartesian symmetry axes of the coils.

We consider a pair of rectangular coils defined by a width, $W$, and a length, $L$, separated by a spacing, $S$ (see Fig. 1 ). The origin of the indicated Cartesian coordinate system is placed at the center of the rectangular volume defined by the coils. The current flowing through either coil rotates such that $\vec{B}(x=y=z=0)$ $=-B_{o_{y}} \hat{y}$, where $B_{o_{y}}$ is positive. We consider our coils to be ideally thin, meaning that the current has no spatial extent outside the line along which it is carried. The expression for the field is obtained using the Biot-Savart law and has been published previously. ${ }^{2}$ This expression is differentiated twice with respect to the $y$-coordinate, and the Helmholtz separation is determined numerically.

Figure 2 shows how $S_{H}$ varies with $R=L / W$. For $R=1$, the spacing is equal to the well-known Helmholtz spacing for a square coil pair: ${ }^{4} S_{H} / W=0.54$. As $R$ increases to infinity, $S_{H} / W$ has an asymptotic limit equal to $1 / \sqrt{3}$, corresponding to four straight infinite wires at $^{4} x= \pm W / 2 ; y= \pm W /(2 \sqrt{3})$.

The functional form of the two curves in Fig. 2 can be understood qualitatively from simple magnetostatic considerations. We focus on the $S_{H} / W$ curve; the $S_{H} / L$ curve can be understood in a similar fashion. Our analysis considers the functional shape of $B_{y}$ along the $y$-axis; see Fig. 3 that considers the simple case of $R=1$, which results in $S_{H} / W=0.54$. When $S<S_{H}$, the field can 


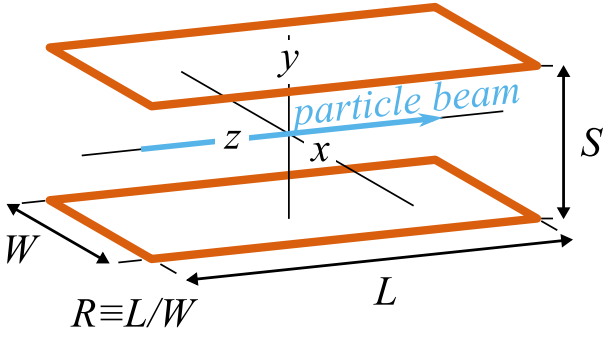

FIG. 1. Rectangular coils and their coordinate system. Throughout this paper, we will assume that the width $W$ remains fixed and alter the length $L$ to obtain different rectangle aspect ratios, $R \equiv L / W$. The spacing between the coils is $S$.

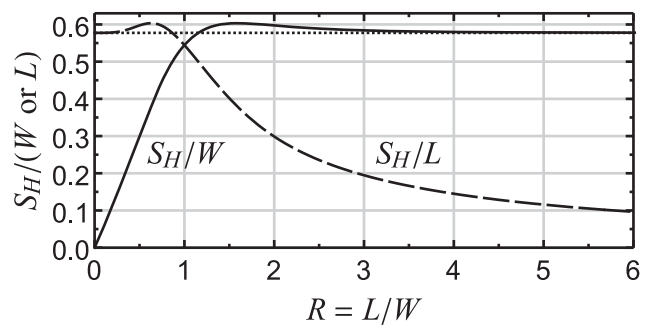

FIG. 2. The Helmholtz separation, $S_{H}$, as a function of the aspect ratio $R$. The same set of spacings is shown in terms of two units represented by the two labeled curves. The solid line shows the spacing in units of the fixed $W$, while the dashed line shows the spacing in units of $L$, which varies with $R$. The dotted line indicates $S_{H}$ for a set of four infinite wires with $x$-spacing $W$.

be described as having a relatively sharp peak at the origin. When $S=S_{H}$, the Helmholtz condition is met and the curve has a relatively broad "plateaued" peak. When $S>S_{H}$, a local minimum, symmetrically centered between two maxima, develops.

Now at $R=\infty$ and $S=S_{H}$, the field shape is plateaued and is due entirely to the four wires parallel to the $z$-axis, the $L$ sides of the rectangle. As these wires are shortened and $R$ retreats from infinity, the four wire segments parallel to the $x$-axis begin to contribute to $B_{y}$ at the origin, causing its functional form to develop a peak there.

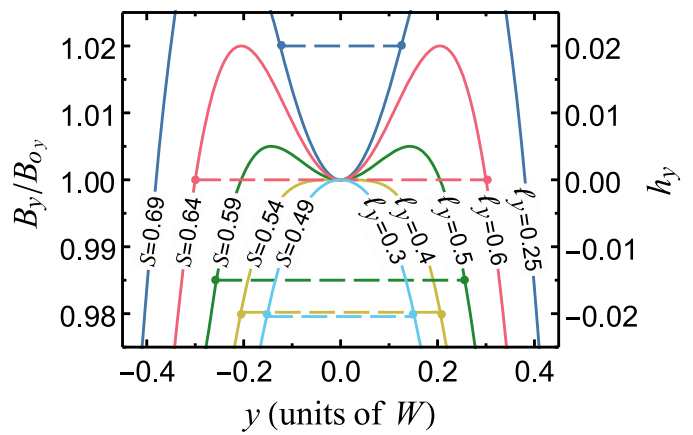

FIG. 3. The values of $B_{y} / B_{o_{y}}$ and $h_{y}$ [see Eq. (1)] along the $y$-axis for a variety of coil spacings when $R=1$. All spacings are in units of $W$. The spacing $S=0.54$ represents the Helmholtz spacing, $S_{H}$. At $S=0.64=S_{y}(0.01)$, the coils are at the "optimum length" spacing; the dashed lines represent the length $\ell_{y}$ within tolerance $T=0.01$ (see text).
In order to restore the plateau shape associated with the Helmholtz condition, the $y$-spacing of the $z$-segments must be increased beyond its asymptotic value so that their (now) two-peaked contribution will serve to compensate the increasingly important end-segment contribution near the origin. Thus, as $R$ decreases from infinity, $S_{H}$ must increase, meaning that there must be a negative slope in the near-asymptotic region of $R$.

At the opposite extreme, as $L$ approaches zero, the geometry is the same as that for the large- $R$ case, but is rotated about the $y$-axis by $90^{\circ}$. That is, when approaching zero, $L$ plays the role that $W$ played when $R$ was approaching infinity. This means that $S_{H}$ and $L$ will be of the same order. Therefore, $S_{H} / W$ must linearly approach zero as $L$ approaches zero, with a positive slope of $1 / \sqrt{3}$. These two slope requirements at $L \ll W$ and $L \gg W$ dictate a local maximum in the intermediate region. This occurs at $R=1.6$ and $S_{H}=0.60$.

At some $R$ value, the short sides of the rectangle are sufficiently distant that in considering the variation in the magnetic field at the origin, one can ignore the short side's contribution and assume that the system consists of four infinite wires. When $R=3.1, S_{H}$ is only $1 \%$ different from its asymptotic value. When $R=5.9, S_{H}$ differs from its asymptotic value by only $0.1 \%$. These two aspect ratios are good reference points when considering if the contributions from the ends of the coils are sufficiently small to neglect their effects.

Previous researchers ${ }^{5,6}$ introduced the idea that the length over which the field is constant within a given tolerance can be extended by increasing the coil spacing beyond $S_{H}$. As discussed above, increasing the spacing from $S_{H}$ introduces a "ripple" in $B_{y}$ along the $y$ axis with a local minimum at the center of the coils (Fig. 3). We follow others ${ }^{7}$ and quantify the variation of the field with the "heterogeneity," $h_{y}$, given by

$$
h_{y}=\frac{B_{y}-B_{o_{y}}}{B_{o_{y}}},
$$

where $B_{y}$ is the $y$-component of the magnetic field at an arbitrary point on one of the three Cartesian axes and $B_{o_{y}}$ is the same at the origin.

We are interested in the length over which $B_{y}$ stays relatively constant. We thus define a length, $\ell_{i}(T)$, along a given axis $i$, which is the longest unbroken distance along that axis, including the origin, over which the range of values of $h_{y}$ is $\leq 2 T$. Values of $\ell_{y}(0.01)$ are shown for a number of coil spacings in Fig. 3. Note that at $S=0.59$, the length includes both positive and negative deviations of $h_{y}$. We define the "optimum length" spacing, $S_{i}(T)$, as the spacing of the coils at which $\ell_{i}(T)$ reaches its maximum, $\ell_{i, \max }(T)$. The optimum length spacing for $T=0.01$ on the $y$-axis, $S_{y}(0.01)$, is represented in Fig. 3 by the $S=0.64$ curve, for which $\ell_{y}(0.01)=\ell_{y, \max }=0.6 \mathrm{~W}$.

Values of $S_{y}(T)$ and $\ell_{y, \max }(T)$ are shown for $T$ values of 0.001 , 0.01 , and 0.1 in Fig. 4. Note that the curves have a similar shape to that for $S_{H} / W$ in Fig. 2. Two important changes in $S_{y}$ and $\ell_{y, \max }$ occur as $T$ increases. First, the maximum value of $S_{y}$ occurs at increasing $R$ values, from $R=1.6(T=0.001)$ to $R=2.2(T=0.1)$. Second, for $T=0.001, \ell_{y, \max }$ is just over half the value of $S_{y}$ for all values of $R$, whereas for $T=0.1, \ell_{y, \max }$ exceeds $S_{y}$ by $40 \%$ at all $R$ values.

Up to this point, we have only considered the uniformity of $B_{y}$ along the $y$-axis. In charged-particle beam experiments, however, the 


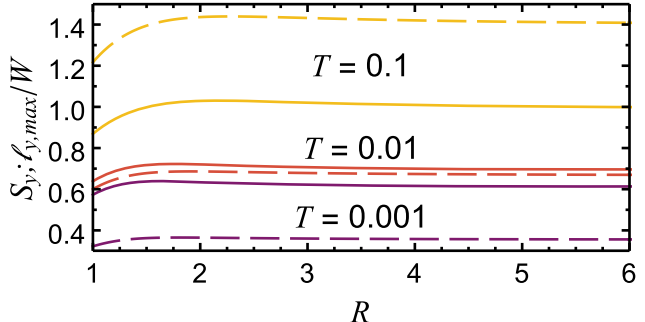

FIG. 4. The "optimum length" spacing for the $y$-axis, $S_{y}(T)$ (solid lines), and the corresponding maximum length of uniform field, $\ell_{y, \max }(T)$ (dashed lines), for rectangular coils, both in units of $W$.

uniformity of $B_{y}$ along the beam path may be more important. Most often, this path will be coincident with the $z$-axis of the rectangular coils. We investigated $S_{i}(T)$ for $B_{y}$ on the $y$-axis and $z$-axis as a function of $R$. The results for $T=0.01$ are shown in Fig. 5 .

A feature of interest in this graph is the $R$ value at which $S_{z}$ and $S_{y}$ are equal. At this point, there is a discontinuity in the values of $\ell_{z}$ and $\ell_{y}$ for $S_{y}$ and $S_{z}$ spacings, respectively. These discontinuities are shown in Figs. 5(b) and 5(c). The discontinuity occurs at this point because $S$ extends beyond the optimum length spacing and the
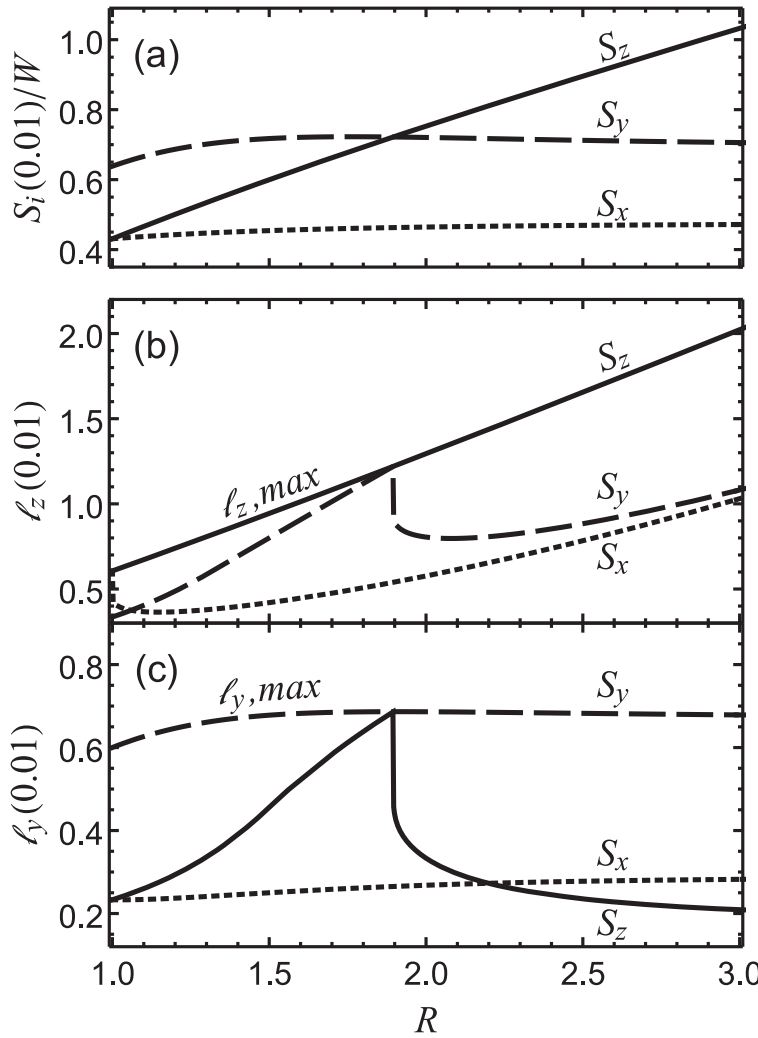

FIG. 5. (a) Coil spacing, $S_{i}(0.01)$, that provides the optimum length, $\ell_{i, \max }(0.01)$, along the desired axis $i ;[(b)$ and (c)] length over which the field is constant to within $T=0.01$ in the $z$-direction and $y$-direction, respectively, for the three optimal length coil spacings shown in (a). terminus of the length over which the field is within tolerance jumps from being outside the location of the peaks in $B_{y}$ to being located just inside the location of the peaks. Such a transition can be seen in Fig. 3 as $S$ moves from $S=0.64$ to $S=0.69$. The value of $R$ at which this transition occurs is a function of $T$. When $T=0$, the crossing occurs at $R=1$. It increases roughly linearly until $T=0.03$, where the crossing happens at $R=3.5$. The value of $R$ where the crossing occurs then quickly increases. At $T=0.05$, the curves cross at $R=10.5$.

Figure 5 raises other important points to consider when designing rectangular coils to minimize magnetic fields in the beamline and target regions. For fixed $W$, the uniform region along the $z$-axis can be extended arbitrarily by increasing $S_{z}$ linearly with $L$. This can become problematic, especially when $R$ exceeds 1.9 (for the case of $T=0.01)$. First, the strength of the field along the beamline decreases steadily as $S_{z}$ is increased, which for sufficiently large $R$ may require unacceptably high currents or power dissipation to "buck out" the local field. Second, as $R$ increases above $1.9, \ell_{y}$ drops significantly for the $S_{z}$ spacing, which may lead to an unacceptable reduction in the volume of a uniform magnetic field in an extended vertical target region.

While the utility of the results discussed here is generally applicable, we now, as an example, consider a set of coils that we have designed for use in our laboratory. They have dimensions $L=1.83 \mathrm{~m}$ and $W=0.81 \mathrm{~m}$, so $S_{H}$ is $0.48 \mathrm{~m}$. The apparatus shielded by these coils has an electron beam line on the $z$-axis that extends from $z=-0.56 \mathrm{~m}$ to $z=+0.15 \mathrm{~m}$, with the target at the origin. The $z$ axis is oriented along Earth's magnetic North-South axis, and the magnetic field in this region has a vertical component of $36 \mu \mathrm{T}$, requiring 22 amp-turns to zero it out at the target center. For a nominal current of $1 \mathrm{~A}$, this corresponds to an array of wires in a square bundle $\sim 0.5 \mathrm{~cm}$ on a side. Our calculations indicate that this nonideally thin set of coils produces a field that is just $0.6 \%$ different at the center than that predicted by our single wire calculation for an equal number of amp-turns. In addition, a reduction in the coil spacing of about $0.3 \%(\sim 2 \mathrm{~mm})$ is required to maintain the Helmholtz condition.

This work was supported by the National Science Foundation (Grant No. PHY-1806776).

\section{DATA AVAILABILITY}

The data that support the findings of this study are available within the article. Additionally, the data are available in tabular form in Zenodo.

\section{REFERENCES}

${ }^{1}$ X. Jing and W. Guo, Chin. J. Mech. Eng. 32, 63 (2019).

${ }^{2}$ F. K. Harris, J. Res. Natl. Bur. Stand. 13, 391 (1934).

${ }^{3}$ M. S. Hao, K. Xiang, Q. Huang, W. M. Sun, and J. H. Zhang, Adv. Mater. Res. 760-762, 1306 (2013)

${ }^{4}$ A. O. Rankine, Proc. Phys. Soc. 46, 1 (1934).

${ }^{5}$ F. R. Crownfield, Rev. Sci. Instrum. 35, 240 (1964).

${ }^{6}$ M. E. Rudd and J. R. Craig, Rev. Sci. Instrum. 39, 1372 (1968).

${ }^{7}$ F. J. Nieves, A. Bayon, and F. Gascon, Rev. Sci. Instrum. 90, 045120 (2019).

${ }^{8}$ K. J. Ahrendsen (2020). "Optimum spacing of thin rectangular magnetic field coils,” Zenodo. https://doi.org/10.5281/zenodo.3960902 